\title{
Convective Diffusion of an Enzyme Reaction
}

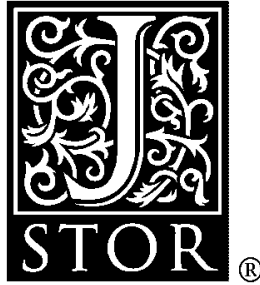

\author{
A. C. Fowler
}

SIAM Journal on Applied Mathematics, Vol. 33, No. 2. (Sep., 1977), pp. 289-297.

Stable URL:

http://links.jstor.org/sici?sici=0036-1399\%28197709\%2933\%3A2\%3C289\%3ACDOAER\%3E2.0.CO\%3B2-4

SIAM Journal on Applied Mathematics is currently published by Society for Industrial and Applied Mathematics.

Your use of the JSTOR archive indicates your acceptance of JSTOR's Terms and Conditions of Use, available at

http://www.jstor.org/about/terms.html. JSTOR's Terms and Conditions of Use provides, in part, that unless you have obtained prior permission, you may not download an entire issue of a journal or multiple copies of articles, and you may use content in the JSTOR archive only for your personal, non-commercial use.

Please contact the publisher regarding any further use of this work. Publisher contact information may be obtained at http://www.jstor.org/journals/siam.html.

Each copy of any part of a JSTOR transmission must contain the same copyright notice that appears on the screen or printed page of such transmission.

JSTOR is an independent not-for-profit organization dedicated to and preserving a digital archive of scholarly journals. For more information regarding JSTOR, please contact support@ jstor.org. 


\title{
CONVECTIVE DIFFUSION OF AN ENZYME REACTION*
}

\author{
A. C. FOWLER $†$
}

\begin{abstract}
The evolution of a simple enzyme reaction being convected by Poiseuille flow in a semi-infinite tube is considered. When the effects of diffusion are ignored, the solutions for the concentrations of enzyme and substrate are analogues of the spatially independent case. When small but nonzero diffusion coefficients are admitted the solutions are modified by the smoothing out of any discontinuities present in the conditions at the inlet to the tube. Explicit solutions in each case are given when the nondimensional concentration of the substrate at the inlet, $f(t)$, is the Heaviside step function

$$
f(t)= \begin{cases}1, & t>0 \\ 0, & t<0\end{cases}
$$
\end{abstract}

1. Introduction. In experiments currently being carried out by Kuchel [4], the evolution of reactions between enzymes and substrates in a convecting fluid is being studied. A fluid flows slowly through a thin tube of radius $\sim 1$ millimetre and length $\sim 1$ metre. The entrance to the tube is connected to two inlets which supply the fluid with enzyme and substrate respectively. The experiment is started with the enzyme inlet open, so that there is a uniform enzyme concentration along the length of the tube, and then at some initial instant the substrate inlet is opened, so that the substrate concentration there is a specified function of time. Reaction between the substrate and enzyme occurs as the reactants are convected with the fluid. In general diffusion also takes place, but the effect of this is usually small for the time scales involved in the experiment [2].

In the system described above, where we have one substrate and one enzyme, the reaction is given by

$$
S+E \underset{k_{-1}}{\stackrel{k_{1}}{\rightleftharpoons}} \stackrel{k_{2}}{\rightarrow} E+P .
$$

Here $S, E, C, P$, stand for substrate, enzyme, complex and product respectively, and $k_{1}, k_{-1}, k_{2}$ are rate constants. The observed result is of the concentration of the product formed as a function of time at a specified distance along the tube. Experimentally, this is found by using a reaction which gives a photosensitive product, so that the concentration is easily found spectrophotometrically. Note that what is actually observed is an integral over volume and time of the concentration, since the photometer is of a finite length and takes a finite time to record an observation; however these may be made so short in practice that the observed result is proportional to the integral of the concentration over the cross-section of the tube.

In this paper we propose and analyze a model system for this experiment and give analytic results which may be used practically.

2. Mathematical model. We consider the fluid to be in fully-formed Poiseuille flow through a semi-infinite cylindrical tube $0<x<\infty, 0<r<a$, so that the velocity in $(x, r)$ coordinates is $\mathbf{v}=\left(k\left(a^{2}-r^{2}\right), 0\right)$ where $k=2 M /\left(\pi \rho a^{4}\right), \rho$ is

\footnotetext{
* Received by the editors August 17, 1976.

$\dagger$ Mathematics Institute, Oxford University, Oxford, England. This work was supported by a grant from the Department of Education of Northern Ireland.
} 
the density and $M$ is the mass flow. At each point of the fluid we denote for convenience the concentration of each reactant by its letter in square brackets. Then the law of mass action applied to (1) gives the following rate equations:

$$
\begin{aligned}
& \frac{\partial[S]}{\partial t}+(\mathbf{v} \cdot \nabla)[S]=D_{s} \nabla^{2}[S]+k_{-1}[C]-k_{1}[S][E], \\
& \frac{\partial[E]}{\partial t}+(\mathbf{v} \cdot \nabla)[E]=D_{e} \nabla^{2}[E]+\left(k_{-1}+k_{2}\right)[C]-k_{1}[S][E], \\
& \frac{\partial[P]}{\partial t}+(\mathbf{v} \cdot \nabla)[P]=D_{p} \nabla^{2}[P]+k_{2}[C], \\
& \frac{\partial[C]}{\partial t}+(\mathbf{v} \cdot \nabla)[C]=D_{c} \nabla^{2}[C]+k_{1}[S][E]-\left(k_{-1}+k_{2}\right)[C] .
\end{aligned}
$$

The second and third term in each equation represent the effects of convection and diffusion, and $D_{s}, D_{e}, D_{p}, D_{c}$ are the respective diffusion coefficients of $S, E, P$ and $C$. Physically $D_{e} \simeq D_{c}, D_{p} \simeq D_{s}$, so we take $D_{e}=D_{c}=D_{2}, D_{p}=D_{s}=D_{1}$. The initial and boundary conditions for (2)-(5) are

$$
\begin{aligned}
& {[S]=[C]=[P]=0, \quad[E]=E_{0} \quad \text { on } t=0, \quad x>0 ;} \\
& {[S]=S_{0} f_{1}(t), \quad[E]=E_{0}, \quad[P]=[C]=0 \quad \text { on } t>0, \quad x=0 .}
\end{aligned}
$$

In (6a) $E_{0}$ is a given constant, and $S_{0}$ is such that $f_{1}$ is $O(1)$. (For example $f_{1} \rightarrow 1$ as $t \rightarrow \infty$.) The condition of no flux through the cylindrical boundary $r=a$ requires

$$
\frac{\partial[S]}{\partial r}=\frac{\partial[C]}{\partial r}=\frac{\partial[P]}{\partial r}=\frac{\partial[E]}{\partial r}=0 \quad \text { on } r=a .
$$

If we add (3) and (5) we get

$$
\frac{\partial}{\partial t}\{[E]+[C]\}+(\mathbf{v} \cdot \nabla)\{[E]+[C]\}=D_{2} \nabla^{2}\{[E]+[C]\}
$$

which on using (6) gives the usual enzyme conservation law, namely

$$
[E]+[C]=E_{0}
$$

Eliminating $[E]$ in $(5)$, we obtain

$$
\frac{\partial[C]}{\partial t}+(\mathbf{v} \cdot \nabla)[C]=D_{2} \nabla^{2}[C]+k_{1}[S]\left\{E_{0}-[C]\right\}-\left(k_{-1}+k_{2}\right)[C] .
$$

We now nondimensionalize by writing

(10) $k_{1} E_{0} t=\bar{t}, \quad r=a \bar{r}, \quad x=\frac{k a^{2}}{k_{1} E_{0}} \bar{x}, \quad[S]=S_{0} s, \quad[C]=E_{0} c, \quad[P]=S_{0} p$. 
Then (9), (2) and (4) become (on dropping the bars on $\bar{t}, \bar{x}, \bar{r}$ for convenience)

$$
\begin{gathered}
\varepsilon \frac{\partial c}{\partial t}+\varepsilon\left(1-r^{2}\right) \frac{\partial c}{\partial x}=\frac{1}{P e_{2}}\left[\frac{1}{r} \frac{\partial}{\partial r}\left(r \frac{\partial c}{\partial r}\right)+\sigma^{2} \frac{\partial^{2} c}{\partial x^{2}}\right]+s(1-c)-K_{s} c \\
\frac{\partial s}{\partial t}+\left(1-r^{2}\right) \frac{\partial s}{\partial x}=\frac{1}{P e_{1}}\left[\frac{1}{r} \frac{\partial}{\partial r}\left(r \frac{\partial s}{\partial r}\right)+\sigma^{2} \frac{\partial^{2} s}{\partial x^{2}}\right]+L_{s} c-s(1-c) \\
\frac{\partial p}{\partial t}+\left(1-r^{2}\right) \frac{\partial p}{\partial x}=\frac{1}{P e_{1}}\left[\frac{1}{r} \frac{\partial}{\partial r}\left(r \frac{\partial p}{\partial r}\right)+\sigma^{2} \frac{\partial^{2} p}{\partial x^{2}}\right]+\mu c
\end{gathered}
$$

where

$$
\begin{aligned}
& P e_{1}=\frac{k_{1} E_{0} a^{2}}{D_{1}}, \quad P e_{2}=\frac{k_{1} S_{0} a^{2}}{D_{2}}, \quad \varepsilon=\frac{E_{0}}{S_{0}}, \quad \sigma^{2}=\frac{k_{1}^{2} E_{0}^{2}}{k^{2} a^{2}}, \\
& K_{s}=\frac{k_{-1}+k_{2}}{k_{1} S_{0}}, \quad L_{s}=\frac{k_{-1}}{k_{1} S_{0}}, \quad \mu=K_{s}-L_{s}=\frac{k_{2}}{k_{1} S_{0}} .
\end{aligned}
$$

Typical values of these constants are given in Table 1 [3].

TABLE 1

\begin{tabular}{ll}
\hline \multicolumn{1}{c}{ Constant } & Order of Magnitude \\
\hline$k_{1}\left(\mathrm{Molar}^{-1} s^{-1}\right)$ & $10^{8}$ \\
$k_{2}\left(\mathrm{~s}^{-1}\right)$ & $10^{2}$ \\
$k_{-1}\left(\mathrm{~s}^{-1}\right)$ & $10^{5}$ \\
$E_{0}(\mathrm{Molar})$ & $10^{-8}$ \\
$S_{0}\left(\mathrm{Molar}^{-1}\right.$ & $10^{-2}$ \\
$D_{1}\left(\mathrm{~cm}^{2} \mathrm{~s}^{-1}\right)$ & $10^{-5}$ \\
$D_{2}\left(\mathrm{~cm}^{2} s^{-1}\right)$ & $10^{-7}$ \\
$a(\mathrm{~cm})$ & $10^{-1}$ \\
$M / \rho\left(\mathrm{cm}^{3} s^{-1}\right)$ & $5 \times 10^{-1}$ \\
\hline
\end{tabular}

With these values

(15) $\varepsilon \sim 10^{-6}, \quad P e_{1} \sim 10^{3}, \quad P e_{2} \sim 10^{11}, \quad \sigma^{2} \sim 10^{-5} ; \quad K_{s}, L_{s} \sim 10^{-1}, \quad \mu \sim 10^{-4}$.

The boundary conditions for (11), (12), (13) are, from (6),

$$
\begin{aligned}
& s=p=c=0 \quad \text { on } t=0, \quad x>0 \\
& p=c=0, \quad s=f(t) \equiv f_{1}\left(\frac{t}{k_{1} E_{0}}\right) \quad \text { on } t>0, \quad x=0 \\
& \frac{\partial s}{\partial r}=\frac{\partial p}{\partial r}=\frac{\partial c}{\partial r}=0 \quad \text { on } r=1 .
\end{aligned}
$$


3. Solution with no diffusion. Since $1 /\left(P e_{1}\right), 1 /\left(P e_{2}\right) \ll 1$, we may as a first approximation neglect diffusion terms in $(11),(12),(13)$ : the equations reduce to

$$
\begin{gathered}
\varepsilon \frac{\partial c}{\partial t}+\varepsilon\left(1-r^{2}\right) \frac{\partial c}{\partial x}=s(1-c)-K_{s} c, \\
\frac{\partial s}{\partial t}+\left(1-r^{2}\right) \frac{\partial s}{\partial x}=L_{s} c-s(1-c), \\
\frac{\partial p}{\partial t}+\left(1-r^{2}\right) \frac{\partial p}{\partial x}=\mu c,
\end{gathered}
$$

together with the boundary conditions (16). Since the effect of diffusion is to "smooth" discontinuities in the solutions, we expect on neglecting it that any discontinuities in the initial conditions (i.e. in $f(t)$ ) will remain in the solution for $t>0$ and be convected with the fluid.

In (17)-(19), $r$ appears only as a parameter, so we may write $y=x /\left(1-r^{2}\right)$, and the equations are

$$
\begin{aligned}
\varepsilon\left(\frac{\partial c}{\partial t}+\frac{\partial c}{\partial y}\right) & =s(1-c)-K_{s} c, \\
\frac{\partial s}{\partial t}+\frac{\partial s}{\partial y} & =L_{s} c-s(1-c), \\
\frac{\partial p}{\partial t}+\frac{\partial p}{\partial y} & =\mu c .
\end{aligned}
$$

Since $\mu=K_{s}-L_{s}$, we may add these and integrate using (16) to obtain

$$
p+s+\varepsilon c= \begin{cases}f(t-y), & y<t, \\ 0, & y>t .\end{cases}
$$

In fact, since the fluid particles in $y>t$ were in $x>0$ at $t=0$, we must have $s=c=p=0$ in $y>0$. Neglecting $O(\varepsilon)$ in (20), we obtain

$$
c=\frac{s}{K_{s}+s} .
$$

This says that the reaction (17), or (5), is in equilibrium and (24) is the usual Michaelis Menten quasi-steady state.

On using (24), (21) becomes

$$
\frac{\partial s}{\partial t}+\frac{\partial s}{\partial y}=\frac{-\mu s}{K_{s}+s}
$$

in $0<y<t$ with boundary condition $s=f(t)$ on $y=0$, the solution of which is given implicitly by

$$
K_{s} \log \left[\frac{s}{f(t-y)}\right]+\{s-f(t-y)\}=-\mu y .
$$


In the special case where $f$ is the Heaviside step function, $s$ may be written explicitly as $s=A(\mu y)$ where $A(r)$ is a modified exponential defined by

$$
A(0)=1, \quad \frac{d A}{d r}=\frac{-A}{K_{s}+A}
$$

again $s=0$ in $y>t$.

Now the solution (24) does not satisfy the boundary condition on $y=0$ (since $s \neq 0$ there), so there must exist a singular region there where the neglected $y$ derivative in (20) becomes important. This is physically of little interest, since the observation is carried out away from $x=0$, but we include an analysis of it for completeness.

If $y=O(\varepsilon)$ and $t=O(1)$ then we put $y=\varepsilon Y$, and obtain

$$
\begin{aligned}
& \varepsilon \frac{\partial c}{\partial t}+\frac{\partial c}{\partial Y}=s(1-c)-K_{s} c \\
& \varepsilon \frac{\partial s}{\partial t}+\frac{\partial s}{\partial Y}=\varepsilon\left[L_{s} c-s(1-c)\right],
\end{aligned}
$$

where we require $c=0$ on $Y=0$ and a matching condition as $Y \rightarrow \infty$. Equation (29) gives $s=f(t)$ to first order, as we expect since there is no boundary layer for $s$, and $f(t)$ is the one term inner expansion of (26) for small $y$. Substituting for $s$ in (28) and neglecting $O(\varepsilon)$, we obtain

$$
\frac{\partial c}{\partial Y}=f(t)(1-c)-K_{s} c, \quad c=0 \text { on } Y=0,
$$

which has solution

$$
c=\frac{f(t)}{K_{s}+f(t)}\left[1-e^{-\left(f(t)+K_{s}\right) Y}\right], \quad t>0 .
$$

This automatically matches with the outer solution. The outer solutions to first order are thus given by (24) and (26), and from (23),

$$
p=f(t-y)-s, \quad y<t .
$$

The concentration per unit length at $x$ is proportional to

$$
\int_{0}^{1} r p\left(\frac{x}{1-r^{2}}, t\right) d r \equiv C(x, t)
$$

Now $p=0$ in $y>t$, i.e. $x /\left(1-r^{2}\right)>t$ or $r>(1-x / t)^{1 / 2}$, so

$$
C(x, t)= \begin{cases}\int_{0}^{(1-x / t)^{1 / 2}} r p d r, & t>x, \\ 0, & t<x .\end{cases}
$$




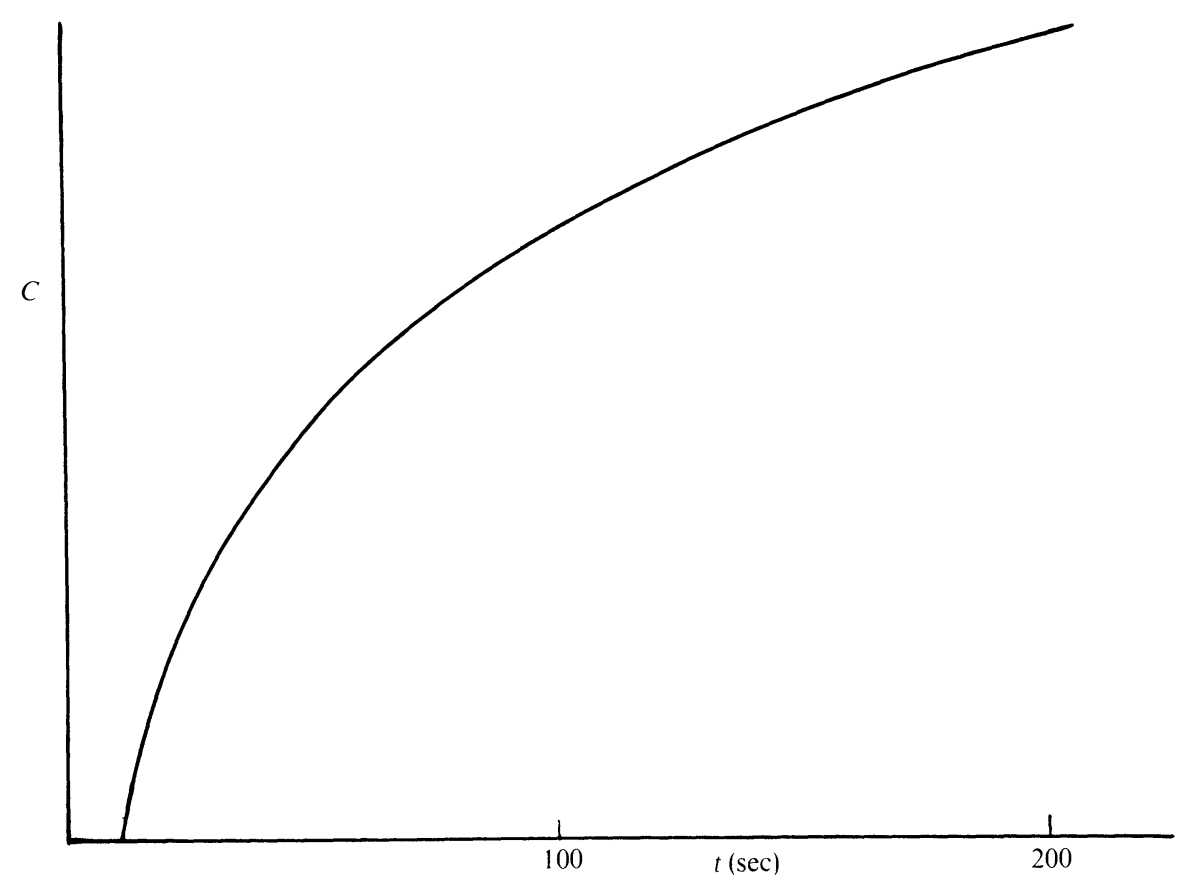

FIG. 1. Response of $C(x, t)$ in arbitrary units to a step function input of substrate at $x=0$, when $a=.05, M / \rho=.12, x=350 \mathrm{~cm}$.

As an example suppose $f(t)$ is the Heaviside step function. Then $s=A(\mu y)$ in $y<t$ and $p=1-A(\mu y)$ whence

$$
C(x, t)=\int_{0}^{(1-x / t)^{1 / 2}} r\left[1-A\left(\frac{\mu x}{1-r^{2}}\right)\right] d r
$$

in $x<t$. From (35),

$$
\begin{array}{rlr}
\frac{\partial C}{\partial t} & =\left(1-\frac{x}{t}\right)^{1 / 2}[1-A(\mu t)] \frac{x}{2 t^{2}}\left(1-\frac{x}{t}\right)^{-1 / 2} & \\
& =\frac{x}{2 t^{2}}[1-A(\mu t)], & x<t
\end{array}
$$

and $C$ is easily computed for any fixed $x$. A typical profile is shown in Fig. 1.

It is clear that in this case the line $x=\left(1-r^{2}\right) t$ is a shock, and in order to determine how dissipation affects the structure of this shock, we must reconsider the diffusion terms in (11), (12) and (13).

4. Solution with diffusion. From Table $1,1 /\left(P e_{2}\right) \ll 1 /\left(P e_{1}\right) \ll 1, \sigma^{2} \ll 1$, so that in the first approximation the dominant diffusion terms in (11), (12), (13) are those corresponding to radial diffusion of $S$ and $P$. If we neglect other diffusion 
terms, we have

$$
\begin{aligned}
\varepsilon \frac{\partial c}{\partial t}+\varepsilon\left(1-r^{2}\right) \frac{\partial c}{\partial x} & =s(1-c)-K_{s} c, \\
\frac{\partial s}{\partial t}+\left(1-r^{2}\right) \frac{\partial s}{\partial x} & =\frac{1}{P e_{1}} \frac{1}{r} \frac{\partial}{\partial r}\left(r \frac{\partial s}{\partial r}\right)+L_{s} c-s(1-c), \\
\frac{\partial p}{\partial t}+\left(1-r^{2}\right) \frac{\partial p}{\partial x} & =\frac{1}{P e_{1}} \frac{1}{r} \frac{\partial}{\partial r}\left(r \frac{\partial p}{\partial r}\right)+\mu c,
\end{aligned}
$$

where $\varepsilon \ll 1,1 /\left(P e_{1}\right) \ll 1, \mu \ll 1$. Solutions of (37)-(39) are required which satisfy the boundary conditions (16) where $f(t)$ is the Heaviside step function.

We wish to examine the behavior near the shock $x=\left(1-r^{2}\right) t$, so we introduce new variables

$$
\xi=\left(1-r^{2}\right) t-x, \quad \eta=r, \quad \tau=t .
$$

Then (37) and (38) become

$$
\begin{aligned}
& \varepsilon \frac{\partial c}{\partial \tau}=s(1-c)-K_{s} c \\
& \frac{\partial s}{\partial \tau}=\frac{1}{P e_{1}}\left[4 \eta^{2} \tau^{2} \frac{\partial^{2} s}{\partial \xi^{2}}-4 \eta \tau \frac{\partial^{2} s}{\partial \xi \partial \eta}-2 \tau \frac{\partial s}{\partial \xi}+\frac{1}{\eta} \frac{\partial s}{\partial \eta}+\frac{\partial^{2} s}{\partial \eta^{2}}\right]+L_{s} c-s(1-c)
\end{aligned}
$$

with a similar equation for $p$. .

Now we write $\xi=\left(1 /\left(P e_{1}^{1 / 2}\right)\right) Z$ and take the outer solutions as those of the preceding section, so that

$$
\begin{array}{ll}
c=s=0, & \xi<0, \\
c=\frac{s}{K_{s}+s}, \quad s=A(\mu y)=A\left(\mu \tau-\frac{\mu \xi}{1-\eta^{2}}\right), & \xi>0 .
\end{array}
$$

With $\tau \sim 1, \eta \sim 1$ we obtain $c=s /\left(K_{s}+s\right)+O(\varepsilon)$ from (41), and

$$
\frac{\partial s}{\partial \tau}=4 \eta^{2} \tau^{2} \frac{\partial^{2} s}{\partial Z^{2}}-\frac{\mu s}{K_{s}+s}+O\left(\varepsilon, \frac{1}{P e_{1}^{1 / 2}}\right)
$$

The matching conditions are

$$
\begin{array}{ll}
s \rightarrow 0 & \text { as } Z \rightarrow-\infty, \\
s \rightarrow A(\mu \tau) & \text { as } Z \rightarrow+\infty, \\
s \rightarrow H(Z) & \text { as } \tau \rightarrow 0,
\end{array}
$$

since the inlet substrate concentration is the Heaviside step function $H(t)$. Finally since $\mu \ll 1$ we can use the method of multiple scales [1] on (44). Formally we introduce a slow time $\tilde{\tau}=\mu \tau$ and a fast time $\tau^{*}=\tau$ and expand $s$ as

$$
s(Z, \tau)=s^{(0)}\left(Z, \tau^{*}, \tilde{\tau}\right)+\mu s^{(1)}\left(Z, \tau^{*}, \tilde{\tau}\right)+\cdots .
$$


Then $s^{(0)}$ satisfies

$$
\begin{array}{cc}
\frac{\partial s^{(0)}}{\partial \tau^{*}}=4 \eta^{2} \tau^{* 2} \frac{\partial^{2} s^{(0)}}{\partial Z^{2}}, \\
s^{(0)} \rightarrow 0 & \text { as } Z \rightarrow-\infty, \\
s^{(0)} \rightarrow A(\tilde{\tau}) & \text { as } Z \rightarrow+\infty, \\
s^{(0)} \rightarrow H(Z) & \text { as } \tau^{*}, \tilde{\tau} \rightarrow 0 .
\end{array}
$$

The solution of (47) is

$$
s^{(0)}=\frac{A(\tilde{\tau})}{\sqrt{\pi}} \int_{-\infty}^{\sqrt{3} Z /\left(4 \eta \tau^{* 3 / 2}\right)} e^{-\theta^{2}} d \theta .
$$

Rewriting this in the original variables and putting $\tilde{\tau}=\mu y$ (since $y \sim \tau$ in the shock) we obtain a uniformly valid first order solution of (37) and (38) as

$$
\begin{aligned}
& s=\frac{A(\mu y)}{\sqrt{\pi}} \int_{-\infty}^{P e_{1}^{1 / 2 \sqrt{3}}\left[\left(1-r^{2}\right) t-x\right] /\left(4 r t^{3 / 2}\right)} e^{-\theta^{2}} d \theta+O\left(\varepsilon, \frac{1}{P e_{1}^{1 / 2}}, \mu\right), \\
& c=\frac{s}{K_{s}+s} .
\end{aligned}
$$

This analysis is invalid when $r \ll 1$, i.e. at the nose of the shock where the diffusion is not dominated by its radial component, but in practice this restriction is not important.

Finally, adding (37), (38), (39) and writing $q=p+s$, we obtain

$$
\frac{\partial q}{\partial \tau}+\varepsilon \frac{\partial c}{\partial \tau}=\frac{1}{P e_{1}} \frac{1}{r} \frac{\partial}{\partial r}\left(r \frac{\partial q}{\partial r}\right)
$$

in the shock we use (40) and $\left.\xi=\left(1 / P e_{1}^{1 / 2}\right)\right) Z$ to get

$$
\begin{array}{cl}
\frac{\partial q}{\partial \tau}=4 \eta^{2} \tau^{2} \frac{\partial^{2} q}{\partial Z^{2}}+O\left(\varepsilon, \frac{1}{P e_{1}^{1 / 2}}\right), \\
q \rightarrow 1 & \text { as } Z \rightarrow+\infty, \\
q \rightarrow 0 & \text { as } Z \rightarrow-\infty, \\
q \rightarrow H(Z) & \text { as } \tau \rightarrow 0 .
\end{array}
$$

The solution of $(51)$ is

$$
q=\frac{1}{\sqrt{\pi}} \int_{-\infty}^{\sqrt{3} Z /\left(4 \eta \tau^{3 / 2}\right)} e^{-\theta^{2}} d \theta
$$

which combined with (48) gives

$$
p=\frac{\left[1-A\left\{\mu x /\left(1-r^{2}\right)\right\}\right]}{\sqrt{ } \pi} \int_{-\infty}^{\sqrt{3} P e_{1}^{1 / 2}\left[\left(1-r^{2}\right) t-x\right] /\left(4 r^{3 / 2}\right)} e^{-\theta^{2}} d \theta+O\left(\varepsilon, \frac{1}{P e_{1}^{1 / 2}}, \mu\right)
$$


and in this case

$$
C(x, t)=\int_{0}^{1} r p d r, \quad x<t
$$

Acknowledgment. I should like to thank Dr. Philip Kuchel for helpful discussions during the course of this work.

\section{REFERENCES}

[1] J. D. Cole, Perturbation Methods in Applied Mathematics, Blaisdell, Waltham, MA, 1968.

[2] J. A. ILlingWORTH AND K. F. TIPTON, An automatic apparatus for the study of enzyme kinetics, Biochem. J., 115 (1969), pp. 511-515.

[3] P. W. KUCHEL, private communication.

$[4] \longrightarrow$, to appear. 